\title{
Minimum generalized degree distance of $n$-vertex tricyclic graphs
}

\section{Asma Hamzeh, Ali Iranmanesh ${ }^{*}$ and Samaneh Hossein-Zadeh}

"Correspondence:

Iranmanesh@modares.ac.ir Department of Mathematics,

Faculty of Mathematical Sciences, Tarbiat Modares University, P.O. Box 14115-137, Tehran, Iran

\begin{abstract}
In (Hamzeh et al. in Stud. Univ. Babeş-Bolyai, Chem., 4:73-85, 2012), we introduced a generalization of a degree distance of graphs as a new topological index. In this paper, we characterize the $n$-vertex tricyclic graphs which have the minimum generalized degree distance.

MSC: 05C12;05C35; 05C05
\end{abstract}

Keywords: generalized degree distance; tricyclic graphs; Harary index; join; extremal graphs

\section{Introduction}

Topological indices and graph invariants based on the distances between the vertices of a graph are widely used in theoretical chemistry to establish relations between the structure and the properties of molecules. They provide correlations with physical, chemical and thermodynamic parameters of chemical compounds [1-3]. The Wiener index is a well-known topological index, which equals to the sum of distances between all pairs of vertices of a molecular graph [4]. It is used to describe molecular branching and cyclicity and establish correlations with various parameters of chemical compounds. In this paper, we only consider simple and connected graphs. Let $G$ be a connected graph with the vertex and edge sets $V(G)$ and $E(G)$, respectively, and the number of vertices and edges of $G$ are denoted, respectively, by $n$ and $m$. As usual, the distance between the vertices $u$ and $v$ of $G$ is denoted by $d_{G}(u, v)(d(u, v)$ for short). It is defined as the length of any shortest path connecting $u$ and $v$ in $G$. We let $d_{G}(v)$ be the degree of a vertex $v$ in $G$. The eccentricity of $v$, denoted by $\varepsilon(v)$, is the maximum distance from vertex $v$ to any other vertex. The diameter of a graph $G$ is denoted by $\operatorname{diam}(G)$ and is the maximum eccentricity over all vertices in a graph $G$. The radius $r=r(G)$ is defined as the minimum of $\varepsilon(v)$ over all vertices $v \in V(G)$. A connected graph $G$ with $n$ vertices and $m$ edges is called tricyclic if $m=n+2$. The join $G=G_{1}+G_{2}$ of two graphs $G_{1}$ and $G_{2}$ with disjoint vertex sets $V_{1}$ and $V_{2}$ and edge sets $E_{1}$ and $E_{2}$ is the graph union $G_{1} \cup G_{2}$ together with all the edges joining $V_{1}$ and $V_{2}$.

Additively weighted Harary index is defined as follows in [5]

$$
H_{A}(G)=\sum_{\{u, v\} \subseteq V(G)} d^{-1}(u, v)\left(d_{G}(u)+d_{G}(v)\right) .
$$

○2013 Hamzeh et al.; licensee Springer. This is an Open Access article distributed under the terms of the Creative Commons Attribution License (http://creativecommons.org/licenses/by/2.0), which permits unrestricted use, distribution, and reproduction in any medium, provided the original work is properly cited. 
There are two papers $[6,7]$, which introduced a new graph invariant with the name degree distance. It is defined as follows:

$$
D^{\prime}(G)=\sum_{\{u, v\} \subseteq V(G)} d(u, v)\left(d_{G}(u)+d_{G}(v)\right) .
$$

Generalized degree distance is denoted by $H_{\lambda}(G)$ and defined as follows in [8].

For every vertex $x, H_{\lambda}(x)$ is defined by $H_{\lambda}(x)=D^{\lambda}(x) d_{G}(x)$, where $D^{\lambda}(x)=\sum_{y \in V(G)} d^{\lambda}(x$, $y$ ), and to avoid confusion, we denote $H_{\lambda}(x)$ in graph $G$ with $H_{\lambda}(x, G)$. So we have

$$
H_{\lambda}(G)=\sum_{x \in V(G)} H_{\lambda}(x)=\sum_{x \in V(G)} D^{\lambda}(x) d_{G}(x)=\sum_{\{u, v\} \subseteq V(G)} d^{\lambda}(u, v)\left(d_{G}(u)+d_{G}(v)\right),
$$

where $\lambda$ is a real number. If $\lambda=0$, then $H_{\lambda}(G)=4 m$. When $\lambda=1$, this new topological index $H_{\lambda}(G)$ equals the degree distance index (i.e., the Dobrynin or the Schultz index). The properties of the degree distance index were studied in [9-12]. Also, if $\lambda=-1$, then $H_{\lambda}(G)=H_{A}(G)$ (see above). The relation of our new index with other intensely studied indices motivated our present (and future) study.

Throughout this paper, $K_{n}$ and $K_{1, n-1}$ denote the complete and star graphs on $n$ vertices, respectively. Our other notations are standard and taken mainly from $[1,2,13]$.

Extremal graph theory is a branch of graph theory that studies extremal (maximal or minimal) graphs, which satisfy a certain property. Extremality can be taken with respect to different graph invariants, such as order, size or girth. The problem of determining extremal values and corresponding extremal graphs of some graph invariants is the topic of several papers, for example, see [9-12, 14-16].

\section{Main results}

In this paper, we characterize all of $n$-vertex tricyclic graphs which have the minimum generalized degree distance.

It is well known that the natural numbers $d_{1} \geq d_{2} \geq \cdots \geq d_{n} \geq 1$ are the degrees of the vertices of a tree if and only if $\sum_{i=1}^{n} d_{i}=2 n-2[17,18]$. The next lemma characterizes connected tricyclic graphs by their degree sequence.

Lemma 2.1 [19] Let $n \geq 4$ and $G$ be an $n$-vertex tricyclic graph. The integers $d_{1} \geq d_{2} \geq$ $\cdots \geq d_{n} \geq 1$ are the degrees of the vertices of a graph $G$ if and only if

(i) $\sum_{i=1}^{n} d_{i}=2 n+4$,

(ii) at least four of them are greater than or equal to 2 ,

(iii) $\left(d_{1}, d_{2}, \ldots, d_{n}\right) \notin\{(n-1,4,3,2,1, \ldots, 1),(n-1,5,2,2,1, \ldots, 1)\}$ for sufficiently large $n$.

Let $x_{i}$ be the number of vertices of degree $i$ of $G$ for $1 \leq i \leq n-1$. If $d_{G}(v)=k$, then

$$
\begin{aligned}
D^{\lambda}(v)=\sum_{u \in V(G)} d^{\lambda}(u, v) & =\sum_{u \in V(G), d(u, v)=1} d^{\lambda}(u, v)+\sum_{u \in V(G), d(u, v) \geq 2} d^{\lambda}(u, v) \\
& \geq k+2^{\lambda}(n-k-1) \\
& =2^{\lambda} n-k\left(2^{\lambda}-1\right)-2^{\lambda},
\end{aligned}
$$


so

$$
\begin{aligned}
H_{\lambda}(G) & =\sum_{v \in V(G)} d_{G}(v) D^{\lambda}(v) \\
& \geq \sum_{k=1}^{n-1} k x_{k}\left(2^{\lambda} n-k\left(2^{\lambda}-1\right)-2^{\lambda}\right) .
\end{aligned}
$$

We define

$$
F_{\lambda}\left(x_{1}, x_{2}, \ldots, x_{n-1}\right)=\sum_{k=1}^{n-1} k x_{k}\left(2^{\lambda} n-k\left(2^{\lambda}-1\right)-2^{\lambda}\right) .
$$

We obtain the minimum of $F_{\lambda}\left(x_{1}, x_{2}, \ldots, x_{n-1}\right)$ over all integers numbers $x_{1}, x_{2}, \ldots, x_{n-1} \geq 0$, which satisfy one of the conditions of Lemma 2.1.

We rewrite Lemma 2.1 in terms of the notations above as follows.

Lemma 2.2 [19] Let $n \geq 4$ and $G$ be an $n$-vertex tricyclic graph. The integers $x_{1}, x_{2}, \ldots$, $x_{n-1} \geq 0$ are the multiplicities of the degrees of a graph $G$ if and only if

(i) $\sum_{i=1}^{n-1} x_{i}=n$,

(ii) $\sum_{i=1}^{n-1} i x_{i}=2 n+4$,

(iii) $x_{1} \leq n-4$,

(iv) $\left(x_{1}, x_{2}, \ldots, x_{n-1}\right) \notin\{(n-4,1,1,1,0, \ldots, 0,1),(n-4,2,0,0,1,0, \ldots, 0,1)\}$ for sufficiently large $n$.

We denote the set of all vectors $\left(x_{1}, \ldots, x_{n-1}\right)$, which satisfy the conditions above, by $\Delta$. Let $G$ be a connected graph with multiplicities of the degrees $\left(x_{1}, \ldots, x_{n-1}\right)$, and let $m \geq 2$, $p>0, m+p \leq n-2, x_{m} \geq 1$ and $x_{m+p} \geq 1$. Now we consider the transformation of $t_{1}$, which is defined as follows [11]:

$$
\begin{aligned}
t_{1}\left(x_{1}, \ldots, x_{n-1}\right) & =\left(x_{1}^{\prime}, \ldots, x_{n-1}^{\prime}\right) \\
& =\left(x_{1}, \ldots, x_{m-1}+1, x_{m}-1, \ldots, x_{m+p}-1, x_{m+p+1}+1, \ldots, x_{n-1}\right) .
\end{aligned}
$$

We have $x_{i}^{\prime}=x_{i}$ for $i \notin\{m-1, m, m+p, m+p+1\}$ and $x_{m-1}^{\prime}=x_{m-1}+1, x_{m}^{\prime}=x_{m}-1$, $x_{m+p}^{\prime}=x_{m+p}-1, x_{m+p+1}^{\prime}=x_{m+p+1}+1$.

Let $2 \leq m \leq n-2, x_{m} \geq 2$. Now consider the transformation $t_{2}$ defined as follows [11]:

$$
\begin{aligned}
t_{2}\left(x_{1}, \ldots, x_{n-1}\right) & =\left(x_{1}^{\prime}, \ldots, x_{n-1}^{\prime}\right) \\
& =\left(x_{1}, \ldots, x_{m-1}+1, x_{m}-2, x_{m+1}+1, \ldots, x_{n-1}\right) .
\end{aligned}
$$

That is $x_{i}^{\prime}=x_{i}$ for $i \notin\{m-1, m, m+1\}$ and $x_{m-1}^{\prime}=x_{m-1}+1, x_{m}^{\prime}=x_{m}-2, x_{m+1}^{\prime}=x_{m+1}+1$.

Lemma 2.3 Suppose that $\lambda$ is a positive integer number, and consider the set of vectors $\left(x_{1}, \ldots, x_{n-1}\right)$.

(1) If $\left(x_{1}, \ldots, x_{n-1}\right) \in \Delta$, then $t_{1}\left(x_{1}, \ldots, x_{n-1}\right) \in \Delta$, unless $m=2$ and $x_{1}=n-4$,

(2) $F_{\lambda}\left(t_{1}\left(x_{1}, x_{2}, \ldots, x_{n-1}\right)\right)<F_{\lambda}\left(x_{1}, x_{2}, \ldots, x_{n-1}\right)$. 
Proof (1) We can easily see that $\sum_{i=1}^{n-1} x_{i}=\sum_{i=1}^{n-1} x_{i}^{\prime}, \sum_{i=1}^{n-1} i x_{i}=\sum_{i=1}^{n-1} i x_{i}^{\prime}$. If $m=2, x_{1}=n-4$, then $x_{1}^{\prime}>n-4$, and in this case, $t_{1}\left(x_{1}, \ldots, x_{n-1}\right) \notin \Delta$. Now if $x_{1}^{\prime}>n-4$, according to $x_{1} \leq$ $n-4$, we have $m=2$ and $x_{1}=n-4$. Therefore, we conclude that if $\left(x_{1}, \ldots, x_{n-1}\right) \in \Delta$, then $x_{1}^{\prime}>n-4$ if and only if $m=2$ and $x_{1}=n-4$.

(2) With a simple calculation, we have

$$
\begin{aligned}
F_{\lambda}\left(x_{1}, x_{2}, \ldots, x_{n-1}\right)-F_{\lambda}\left(t_{1}\left(x_{1}, x_{2}, \ldots, x_{n-1}\right)\right) & =\left(2^{\lambda}-1\right)(2 p+2) \\
& >0 .
\end{aligned}
$$

The proof is now completed.

Lemma 2.4 Suppose that $\lambda$ is a positive integer number, and consider the set of vectors $\left(x_{1}, \ldots, x_{n-1}\right)$.

(1) If $\left(x_{1}, \ldots, x_{n-1}\right) \in \Delta$, then $t_{2}\left(x_{1}, \ldots, x_{n-1}\right) \in \Delta$, unless $m=2$ and $x_{1}=n-4$,

(2) $F_{\lambda}\left(t_{2}\left(x_{1}, x_{2}, \ldots, x_{n-1}\right)\right)<F_{\lambda}\left(x_{1}, x_{2}, \ldots, x_{n-1}\right)$.

Proof The proof is similar to the proof of the previous lemma, by taking $p=0$.

Theorem 2.5 Let $n \geq 4$ and $\lambda$ be a positive integer. If $G$ belongs to the class of connected tricyclic graphs on $n$ vertices, then we have

$$
\min H_{\lambda}(G)=\left(n^{2}-n+24\right)+2^{\lambda}\left(n^{2}+3 n-28\right)
$$

and all the extremal graphs are isomorphic to $H$ or $H^{\prime}$, where $H$ is obtained by identifying the center of a star $K_{1, n-4}$ with an arbitrary vertex of a complete graph $K_{4}$, and $H^{\prime}$ is obtained by identifying the center of a star $K_{1, n-5}$ with an arbitrary four degree vertex of a graph $A_{2}$, which is shown in Figure 1, respectively.

Proof To find minimum $H_{\lambda}(G)$, over the classes of connected tricyclic graphs with $n$ vertices, it is enough to find $\min _{\left(x_{1}, x_{2}, \ldots, x_{n-1}\right) \in \Delta} F_{\lambda}\left(x_{1}, x_{2}, \ldots, x_{n-1}\right)$. At first, we consider the case $n=4$. In this case, only tricyclic graph with 4 vertices is $K_{4}$, that is, $H_{\lambda}\left(K_{4}\right)=36$, and the theorem is proved in this case.

Now let $n \geq 5$. Let us consider $n=5$. The all connected tricyclic graphs with 5 vertices, is $A_{1}, A_{2}, A_{3}$ and $A_{4}$. Meanwhile, $H_{\lambda}\left(A_{1}\right)=44+3 \cdot 2^{\lambda+2}=\theta(5), H_{\lambda}\left(A_{2}\right)=44+3 \cdot 2^{\lambda+2}=\theta(5)$, where $\theta(n)=\left(n^{2}-n+24\right)+2^{\lambda}\left(n^{2}+3 n-28\right), H_{\lambda}\left(A_{3}\right)=40+2^{\lambda+4}$, and $H_{\lambda}\left(A_{3}\right)=42+2^{\lambda} \cdot 14$, respectively, where $A_{i}(i=1, \ldots, 4)$ are receipted in Figure 1 . So $A_{1}$ and $A_{2}$ are extremal graphs, and the theorem is proved in this case.

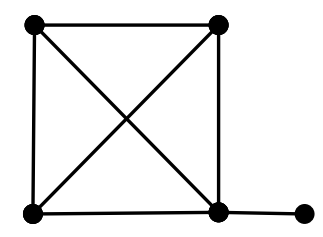

$A_{1}$

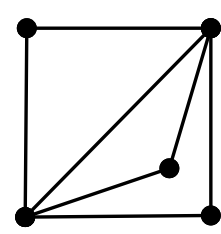

$A_{2}$

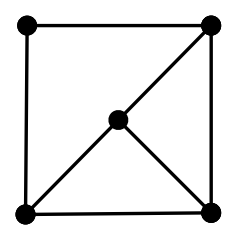

$A_{3}$

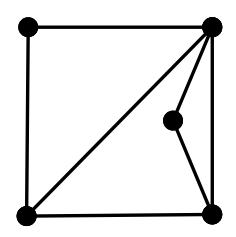

$A_{4}$

Figure 1 The graphs $A_{1}, A_{2}, A_{3}$ and $A_{4}$ 
Finally, let $n \geq 6$. If $x_{n-1} \geq 2$, consider two different vertices $x, y \in V(G)$ such that $d_{G}(x)=$ $d_{G}(y)=n-1$. Since $n \geq 6$, we can choose four different vertices $p, q, r, s \in V(G)-\{x, y\}$. Actually, the vertices $p, q, r, s$ are all adjacent to $x$ and $y$, hence $G$ has at least four cycles $x y p x, x y q x, x y r x$, and $x y s x$, which contradicts the hypothesis. Therefore, $x_{n-1} \leq 1$.

Let us analyze the possible values for $x_{5}, \ldots, x_{n-2}$. If there exist $5 \leq i<j \leq n-2$ such that $x_{i} \geq 1$ and $x_{j} \geq 1$, then, by applying $t_{1}$ for the positions $i$ and $j$, we obtain a new vector $\left(x_{1}^{\prime}, \ldots, x_{n-1}^{\prime}\right) \in \Delta$, for which $F_{\lambda}\left(x_{1}^{\prime}, \ldots, x_{n-1}^{\prime}\right)<F_{\lambda}\left(x_{1}, \ldots, x_{n-1}\right)$. Similarly, if there exists $5 \leq i \leq n-2$ such that $x_{i} \geq 2$, then by $t_{2}$, we obtain a new degree sequence in $\Delta$, for which $F_{\lambda}\left(x_{1}^{\prime}, \ldots, x_{n-1}^{\prime}\right)<F_{\lambda}\left(x_{1}, \ldots, x_{n-1}\right)$, that is, $x_{i}(i=5, \ldots, n-2)$ are not greater than 1 . Let us show that all vectors $\left(x_{1}, \ldots, x_{n-1}\right) \in \Delta$, realizing the minimum of $F_{\lambda}$, have $x_{5}=\cdots=x_{n-2}=0$.

Actually, suppose that there is an index $5 \leq i \leq n-2$ such that $x_{i}=1$ and $x_{k}=0$ for all $5 \leq k \leq n-2, k \neq i$. In this case, if $x_{4} \geq 1$, we can apply $t_{1}$ for positions 4 and $i$ and obtain a smaller value for $F_{\lambda}$. Suppose that $x_{4}=0$. Since $x_{n-1} \in\{0,1\}$, we will separately analyze the two cases: (a) $x_{n-1}=1$ and (b) $x_{n-1}=0$.

(a) In this case, $x_{n-1}=x_{i}=1$, where $i \geq 5$ and $x_{4}=0$. We can consider different vertices $x, y, p, q, r, s \in V(G)$ such that $d_{G}(x)=n-1 \geq 5, d_{G}(y)=i \geq 5, p, q, r, s$, all are adjacent to $x$ and $y$, respectively. Meanwhile, $x$ and $y$ are adjacent. We have found four cycles $x y p x$, $x y q x, x y r x$, and $x y s x$, which contradicts the hypothesis.

(b) If $x_{n-1}=0$, then $x_{3}=0, x_{i}=1(5 \leq i \leq n-2)$, and $\Delta$ is characterized by the equations $x_{1}+x_{2}=n-1, x_{1}+2 x_{2}+i=2 n+4$, and $x_{1} \leq n-8$. Suppose that $x_{3} \geq 1$. In this case, we can use $t_{1}$ for position 3 and $i$ and deduce a smaller value for $F_{\lambda}$. Thus $x_{1}+x_{2}=n-1$ and $x_{1}+2 x_{2}+i=2 n+4$. We can obtain $x_{1}=i-6 \leq n-8$. Moreover, $x_{2}=n-i+5 \geq 7$. In this case, we can use $t_{1}$ for position 2 and $i$ and deduce a smaller value for $F_{\lambda}$.

To sum up, we have $x_{5}=\cdots=x_{n-2}=0$ and $x_{n-1} \in\{0,1\}$. We will separately analyze the two cases: $\left(\mathrm{a}^{\prime}\right) x_{n-1}=0$ and $\left(\mathrm{b}^{\prime}\right) x_{n-1}=1$ again.

$\left(\mathrm{a}^{\prime}\right)$ If $x_{n-1}=0$, then $x_{2}+2 x_{3}+3 x_{4}=n+4$. This equation does not hold. If all $x_{2}, x_{3}$, and $x_{4}$ are not greater than $2, x_{2}+2 x_{3}+3 x_{4} \leq 12$, which contradicts the hypothesis $n \geq 6$. If one of $x_{2}, x_{3}$, and $x_{4}$ is greater than 2 , then by using $t_{2}$ for the corresponding position, we can obtain a smaller value for $F_{\lambda}$.

(b') If $x_{n-1}=1$, then $x_{1}+x_{2}+x_{3}+x_{4}=n-1$ and $x_{1}+2 x_{2}+3 x_{3}+4 x_{4}=n+5$, namely $x_{2}+2 x_{3}+3 x_{4}=6$. If $x_{4} \geq 3$, then $x_{2}+2 x_{3}+3 x_{4} \geq 9$. If $x_{4}=2$, then $x_{2}=x_{3}=0$ and $x_{1}=$ $n-1$, which contradicts the condition (iii) in Lemma 2.2. Thus, $x_{4}=0$ or $x_{4}=1$. We now distinguish the following two subcases:

Subcase 1. If $x_{4}=0$, then $x_{3}=3-\frac{x_{2}}{2}$. All its solutions are $x_{2}=0, x_{3}=3$ or $x_{2}=2, x_{3}=2$ or $x_{2}=4, x_{3}=1$ or $x_{2}=6, x_{3}=0$. In the third and fourth cases, by using $t_{2}$ for the position 2 , we can obtain a smaller value for $F_{\lambda}$. In the second case, by using $t_{2}$ for the position 2 , we obtain the first case. But the first case cannot be changed, since by using $t_{2}$ for the position 3 , the vector $(n-4,0,3,0, \ldots, 0,1)$ can be $(n-4,1,1,1,0, \ldots, 0,1)$, which contradicts Lemma 2.2. Thus, $\left(x_{1}, x_{2}, \ldots, x_{n-1}\right)=(n-4,0,3,0, \ldots, 0,1)$, and the corresponding unique graph is denoted by $H$.

Subcase 2. If $x_{4}=1$, then $x_{2}+2 x_{3}=3$. All its solutions are $x_{2}=1, x_{3}=1$ or $x_{2}=3, x_{3}=0$. The first case should be removed. By a similar reasoning as before, the second case cannot be transferred by $t_{2}$. Meanwhile, the first transformation cannot be used. Otherwise, the sequence can be changed into $(n-4,2,0,0,1,0, \ldots, 0,1)$, which contradicts Lemma 2.2 . 
Thus, $\left(x_{1}, x_{2}, \ldots, x_{n-1}\right)=(n-5,3,0,1,0, \ldots, 0,1)$, and the corresponding unique graph is denoted by $H^{\prime}$.

We can easily obtain $H_{\lambda}(H)$ and $H_{\lambda}\left(H^{\prime}\right)$. Thus, $H_{\lambda}(H)=H_{\lambda}\left(H^{\prime}\right)=\left(n^{2}-n+24\right)+2^{\lambda}\left(n^{2}+\right.$ $3 n-28)$. So, case (b) holds. This completes the proof of Theorem 2.5 .

In [19], the authors proved the following theorem.

Theorem 2.6 Let $n \geq 4$. If $G$ belongs to the class of connected tricyclic graphs on $n$ vertices, then:

(a) If $n=4$, then $\min D^{\prime}(G)=36$ and the unique extremal graph is isomorphic to $K_{4}$;

(b) If $n \geq 5$, then $\min D^{\prime}(G)=3 n^{2}+5 n-32$, and all the extremal graphs are isomorphic to $H$ or $H^{\prime}$, where $H$ is obtained by identifying the center of a star $K_{1, n-4}$ with an arbitrary vertex of a complete graph $K_{4}$, and $H^{\prime}$ is obtained by identifying the center of a star $K_{1, n-5}$ with an arbitrary degree 4 vertex of a graph $A_{2}$, which is shown in Figure 1, respectively.

If we choose $\lambda=1$ in Theorem 2.5, then we can obtain the same result (Theorem 3.1 in [19]).

Theorem 2.7 Let $G$ belong to the class of connected graphs on $n$ vertices, and let $\lambda$ be a positive integer number, then for every $n \geq 2$, we have

$$
\min H_{\lambda}(G)=\left(n^{2}-n\right)+\left(n^{2}-3 n+2\right) 2^{\lambda}
$$

and the unique extremal graph is $K_{1, n-1}$.

Proof Since for $n=2$ the property is trivial, so let $n \geq 3$. If $G$ is a connected graph with $n$ vertices, then $\sum_{i=1}^{n-1} x_{i}=n$ and $\sum_{i=1}^{n-1} i x_{i} \geq 2(n-1)$.

We will find the minimum of $F_{\lambda}\left(x_{1}, x_{2}, \ldots, x_{n-1}\right)$ over all natural numbers $x_{1}, x_{2}, \ldots$, $x_{n-1} \geq 0$ satisfying $\sum_{i=1}^{n-1} x_{i}=n$ and $\sum_{i=1}^{n-1} i x_{i} \geq 2(n-1)$. First, we shall prove that under these conditions, all systems $\left(x_{1}, x_{2}, \ldots, x_{n-1}\right)$ of natural numbers reaching $\min F_{\lambda}\left(x_{1}, x_{2}, \ldots, x_{n-1}\right)$ must satisfy (i) and (ii) given below:

(i) $x_{1}+\cdots+x_{n-1}=n$;

(ii) $x_{1}+2 x_{2}+\cdots+(n-1) x_{n-1}=2 n-2$.

Indeed, suppose that $x_{1}+2 x_{2}+\cdots+(n-1) x_{n-1}>2 n-2$. It follows that there exists a smallest index $m, 2 \leq m \leq n-1$ such that $x_{m}>0$. We shall define the system $\left(y_{1}, \ldots, y_{n-1}\right)$ such that $y_{m-1}=x_{m-1}+1, y_{m}=x_{m}-1$ and $y_{i}=x_{i}$ for every $i \neq m-1, m$. We have $\sum_{i=1}^{n-1} y_{i}=n$, $\sum_{i=1}^{n-1} i y_{i}=\sum_{i=1}^{n-1} i x_{i}-1 \geq 2(n-1)$ and $F_{\lambda}\left(x_{1}, x_{2}, \ldots, x_{n-1}\right)-F_{\lambda}\left(y_{1}, y_{2}, \ldots, y_{n-1}\right)=2^{\lambda}(-2 m+n)+$ $(2 m-1)>0$.

Hence $F_{\lambda}$ cannot be minimum for systems $\left(x_{1}, x_{2}, \ldots, x_{n-1}\right)$, for which $\sum_{i=1}^{n-1} i x_{i}>2(n-1)$. Since natural numbers $d_{1}, \ldots, d_{n}$ are the degrees of the vertices of a tree of order $n$ if and only if $\sum_{i=1}^{n} d_{i}=2 n-2$, we can consider that the numbers $x_{1}, x_{2}, \ldots, x_{n-1}$ are the multiplicities of the degrees $1, \ldots, n-1$ of the vertices of a tree $T$ of order $n$, and we shall denote also $F_{\lambda}(T)$ instead of $F_{\lambda}\left(x_{1}, x_{2}, \ldots, x_{n-1}\right)$. Hence the domain where $F_{\lambda}$ must be minimized is defined by the multiplicities of the degrees $1, \ldots, n-1$ of the vertices of a tree $T$ of order $n$.

If $T$ is not isomorphic to $K_{1, n-1}$, i.e., if $\left(x_{1}, x_{2}, \ldots, x_{n-1}\right) \neq(n-1,0, \ldots, 0,1)$, then $\operatorname{diam}(T) \geq 3$. Let $z, t \in V(T)$ such that $d(z, t)=\operatorname{diam}(T)$. It follows that $d_{T}(z)=d_{T}(t)=1$, 
and denote by $u$ and $v, u \neq v$, the vertices adjacent with $t$ and $z$, respectively. Suppose that $d_{T}(u)=m+p$ and $d_{T}(v)=m$, where $m \geq 2$ and $p \geq 0$.

Let $T_{1}$ be the tree of order $n$ defined in the following way: $V\left(T_{1}\right)=V(T)$ and $E\left(T_{1}\right)=$ $E(T) \cup\{z u\}-\{z v\}$. By replacing $T$ with $T_{1}$, the degrees of $u$ and $v$ change: $d_{T_{1}}(u)=m+p+1$, $d_{T_{1}}(v)=m-1$, and the degrees of $n-2$ vertices remain unchanged. We get $F_{\lambda}(T)-F_{\lambda}\left(T_{1}\right)=$ $\left(2^{\lambda}-1\right)(2 p+2)>0$, and $F_{\lambda}(T)$ cannot be minimum.

It follows that $F_{\lambda}(T)$ is minimum only if $T \cong K_{1, n-1}$, i.e., $x_{1}=n-1, x_{2}=\cdots=$ $x_{n-2}=0$ and $x_{n-1}=1$, when $F_{\lambda}(n-1,0, \ldots, 0,1)=\left(n^{2}-n\right)+\left(n^{2}-3 n+2\right) 2^{\lambda}$. By concluding, $\min F_{\lambda}\left(x_{1}, \ldots, x_{n-1}\right)=\left(n^{2}-n\right)+\left(n^{2}-3 n+2\right) 2^{\lambda}$, and equality holds if and only if $x_{1}=n-1$, $x_{2}=\cdots=x_{n-2}=0$ and $x_{n-1}=1$, i.e., $G \cong K_{1, n-1}$ since $H_{\lambda}(G)=\left(n^{2}-n\right)+\left(n^{2}-3 n+2\right) 2^{\lambda}$.

In [12], the authors proved the following theorem.

Theorem 2.8 Let $G$ belong to the class of connected graphs on $n$ vertices, then for every $n \geq 2$, we have

$$
\min D^{\prime}(G)=3 n^{2}-7 n+4,
$$

and the unique extremal graph is $K_{1, n-1}$.

If we choose $\lambda=1$ in Theorem 2.7, then we can obtain the same result (Theorem 2.1 in [12]).

A Moore graph is a graph of diameter $k$ with girth $2 k+1$. Those graphs have the minimum number of vertices possible for a regular graph with given diameter and the maximum degree. We first bring the following results in [20,21].

Lemma 2.9 [20] Let $G$ be a connected graph of order $n \geq 2$ and size $m \geq 1$, and let $\lambda$ be a negative integer. Then $H_{\lambda}(G) \leq\left(1-2^{\lambda}\right) M_{1}(G)+2^{\lambda+1} m n-2^{\lambda+1} m$, and the equality holds if and only if $d \leq 2$, where $d$ is the diameter of $G$.

Lemma 2.10 [21] Let $G$ be a triangle- and quadrangle-free graph on $n$ vertices with radius $r$. Then $M_{1}(G) \leq n(n+1-r)$. The equality is valid if and only if $G$ is a Moore graph of diameter 2 or $G=C_{6}$.

Theorem 2.11 Let $G$ be a triangle- and quadrangle-free graph on $n$ vertices and $m$ edges with radius $r$, and let $\lambda$ be a negative integer. Then $H_{\lambda}(G) \leq\left(1-2^{\lambda}\right) n(n+1-r)+2^{\lambda+1} m n-$ $2^{\lambda+1} m$. The equality is valid if and only if $G$ is a Moore graph of diameter 2 or $G=C_{6}$.

Proof By the lemmas above, we have

$$
\begin{aligned}
H_{\lambda}(G) & \leq\left(1-2^{\lambda}\right) M_{1}(G)+2^{\lambda+1} m n-2^{\lambda+1} m \\
& \leq\left(1-2^{\lambda}\right) n(n+1-r)+2^{\lambda+1} m n-2^{\lambda+1} m .
\end{aligned}
$$

The first equality holds if and only if the diameter of $G$ is at most 2 , and the second one holds if and only if $G$ is a Moore graph of diameter 2 or $G=C_{6}$. So, the equality holds if and only if $G$ is a Moore graph of diameter 2 or $G=C_{6}$. This completes the proof. 


\section{Competing interests}

The authors declare that they have no competing interests.

\section{Authors' contributions}

All authors read and approved the final manuscript.

\section{Acknowledgements}

The authors would like to thank the referee for the valuable comments.

Received: 1 May 2013 Accepted: 29 August 2013 Published: 19 Nov 2013

\section{References}

1. Diudea, MV, Gutman, I, Jantschi, L: Molecular Topology. Huntington, New York (2001)

2. Trinajstić, N: Chemical Graph Theory. CRC Press, Boca Raton (1992)

3. Todeschini, R, Consonni, V: Handbook of Molecular Descriptors. Wiley, New York (2000)

4. Wiener, H: Structural determination of the paraffin boiling points. J. Am. Chem. Soc. 69, 17-20 (1947)

5. Alizadeh, Y, Iranmanesh, A, Došlić, T: Additively weighted Harary index of some composite graphs. Discrete Math. 313, 26-34 (2013)

6. Dobrynin, AA, Kochetova, AA: Degree distance of a graph: a degree analogue of the Wiener index. J. Chem. Inf. Comput. Sci. 34, 1082-1086 (1994)

7. Gutman, I: Selected properties of the Schultz molecular topological index. J. Chem. Inf. Comput. Sci. 34, 1087-1089 (1994)

8. Hamzeh, A, Iranmanesh, A, Hossein-Zadeh, S, Diudea, MV: Generalized degree distance of trees, unicyclic and bicyclic graphs. Stud. Univ. Babeş-Bolyai, Chem. 4, 73-85 (2012)

9. Dankelmann, P, Gutman, I, Mukwembi, S, Swart, HC: On the degree distance of a graph. Discrete Appl. Math. 157, 2773-2777 (2009)

10. Hossein-Zadeh, S, Hamzeh, A, Ashrafi, AR: Extremal properties of Zagreb coindices and degree distance of graphs. Miskolc Math. Notes 11, 129-137 (2010)

11. Tomescu, I: Unicyclic and bicyclic graphs having minimum degree distance. Discrete Appl. Math. 156, 125-130 (2008)

12. Tomescu, l: Some extremal properties of the degree distance of a graph. Discrete Appl. Math. 98, 159-163 (1999)

13. Harary, F: Graph Theory. Addison-Wesley, Reading (1969)

14. Ashrafi, AR, Došlić, T, Hamzeh, A: Extremal graphs with respect to the Zagreb coindices. MATCH Commun. Math. Comput. Chem. 65, 85-92 (2011)

15. Bucicovschi, O, Cioabă, SM: The minimum degree distance of graphs of given order and size. Discrete Appl. Math. 156, 3518-3521 (2008)

16. Khormali, O, Iranmanesh, A, Gutman, I, Ahmadi, A: Generalized Schultz index and its edge versions. MATCH Commun. Math. Comput. Chem. 64, 783-798 (2010)

17. Moon, JW: Counting Labelled Trees. Canadian Mathematical Monographs, vol. 1. Clowes, London (1970)

18. Senior, JK: Partitions and their representative graphs. Am. J. Math. 73, 663-689 (1951)

19. Zhu, W, Hu, S, Ma, H: A note on tricyclic graphs with minimum degree distance. Discrete Math. Algorithms Appl. 3, 25-32 (2011)

20. Hamzeh, A, Iranmanesh, A, Hossein-Zadeh, S: On the generalization of degree distance of graphs (submitted)

21. Yamaguchi, S: Estimating the Zagreb indices and the spectral radius of triangle-and quadrangle-free connected graphs. Chem. Phys. Lett. 458, 396-398 (2008)

10.1186/1029-242X-2013-548

Cite this article as: Hamzeh et al.: Minimum generalized degree distance of $n$-vertex tricyclic graphs. Journal of Inequalities and Applications 2013, 2013:548

\section{Submit your manuscript to a SpringerOpen ${ }^{\circ}$ journal and benefit from:}

- Convenient online submission

Rigorous peer review

- Immediate publication on acceptance

- Open access: articles freely available online

- High visibility within the field

- Retaining the copyright to your article 